\title{
A halogen-free synthesis of gold nanoparticles using gold(III) oxide
}

\author{
Volodymyr Sashuk $\cdot$ Konrad Rogaczewski
}

Received: 20 May 2016/Accepted: 20 August 2016/Published online: 29 August 2016

(C) The Author(s) 2016. This article is published with open access at Springerlink.com

\begin{abstract}
Gold nanoparticles are one of the most used nanomaterials. They are usually synthesized by the reduction of gold(III) chloride. However, the presence of halide ions in the reaction mixture is not always welcome. In some cases, these ions have detrimental influence on the morphology and structure of resulting nanoparticles. Here, we present a simple and halogen-free procedure to prepare gold nanoparticles by reduction of gold(III) oxide in neat oleylamine. The method provides the particles with an average size below $10 \mathrm{~nm}$ and dispersity of tens of percent. The process of nanoparticle formation was monitored using UV-Vis spectroscopy. The structure and chemical composition of the nanoparticles was determined by SEM, XPS and EDX. We also proposed the mechanism of reduction of gold(III) oxide based on MS, IR and NMR data. Importantly, the synthetic protocol is general and applicable for the preparation of other coinage metal nanoparticles from the corresponding metal oxides. For instance, we demonstrated that the absence of halogen enables efficient alloying of metals when preparing gold-silver bimetallic nanoparticles.
\end{abstract}

Electronic supplementary material The online version of this article (doi:10.1007/s11051-016-3576-x) contains supplementary material, which is available to authorized users.

V. Sashuk $(\bowtie) \cdot$ K. Rogaczewski Institute of Physical Chemistry, Polish Academy of Sciences, 01-224 Warsaw, Poland

e-mail: vsashuk@ichf.edu.pl
Keywords Gold nanoparticles · Gold(III) oxide · Oleylamine $\cdot$ Bimetallic nanoparticles

\section{Introduction}

Gold nanoparticles (AuNPs) — due to chemical robustness, easy preparation and functionalization - are often used as a model system for studying nanoscale phenomena (Daniel and Astruc 2004; Hakkinen 2012; Kalsin et al. 2006; Nealon et al. 2012; Sashuk 2012; Sashuk et al. 2012, 2013; Teulle et al. 2015; Wells et al. 2015; Zhao et al. 2016). Moreover, the pristine chemical and physical properties of nanoscopic gold provide additional appeal for applications such as catalysis or sensing (Giljohann et al. 2010; Jans and Huo 2012; Kale et al. 2014; Mikami et al. 2013; Saha et al. 2012; Stratakis and Garcia 2012; Yeh et al. 2012). AuNPs are routinely synthesized by reducing gold halides, typically gold(III) chloride (Bhargava et al. 2005; Brust et al. 1994; Dozol et al. 2013; Jana et al. 2001; Jana and Peng 2003; Kimling et al. 2006; Lee et al. 2010; Leff et al. 1996; Martin et al. 2010; Perrault and Chan 2009; Zhao et al. 2013). The role of halogen in this process is generally overlooked since the size and shape-two basic parameters of the $\mathrm{NP}$ - can be controlled by reductants and ligands employed (Scarabelli et al. 2014; Zhang et al. 2014). Though, the halide ions present in a solution can affect the morphology and surface chemistry of NPs (Li et al. 2013; Rai et al. 2006; Singh et al. 2007). The presence of 
halides in a reaction mixture can also influence the final composition of NPs, e.g. Au-Ag alloys (Rajendra et al. 2015). Hence, we wondered whether gold oxide could be an alternative to gold chloride. The both gold precursors are commercially available on comparable prices that were an additional reason to pursue the research. Meisel and co-workers have recently shown that gold(III) oxide undergoes decomposition to gold colloids upon reduction by molecular hydrogen (Merga et al. 2010). The method, however, requires a special set-up by virtue of the risk of gas explosion that restricts significantly its practical utility. Also, the as-prepared NPs are quite large (20-100 nm) displaying a moderate degree of monodispersity. From application point of view, the smaller nanoparticles with narrow size distribution would be more desirable because of, e.g. more pronounced intrinsic properties, better resistance against aggregation and suitability for studying self-assembly processes.

Herein, we present a simple and convenient method to obtain gold nanoparticles by reduction of gold(III) oxide with oleylamine. Remarkably, the amine (Mourdikoudis and Liz-Marzán 2013; Yu et al. 2014) acts as all-in-one reagent with functions of reductant, ligand and reaction medium. The reaction yields NPs with a small mean size (5-9 $\mathrm{nm}$ ) and reasonable polydispersity $(<1.5)$. Thus, the method offers a specific size range not accessible from classical oleylamine reduction route based on gold(III) chloride. For instance, the NPs obtained under solution processing conditions were usually above $9 \mathrm{~nm}$ (Aslam et al. 2004; Fanizza et al. 2013; Hiramatsu and Osterloh 2004; Lakshminarayana and Qing-Hua 2009; Shen et al. 2008). On other hand, mechanochemical synthesis performed in bulk afforded ultra-small particles with diameters between 1 and $5 \mathrm{~nm}$ (Rak et al. 2014). The use of gold(III) oxide affects not only the size of NPs but also enables to preserve the initial metal ratio when preparing $\mathrm{Au}-\mathrm{Ag}$ alloy NPs. In contrast, the alloys obtained from gold(III) chloride are characterized by reduced silver content owing to solubility issues.

\section{Experimental}

\section{Materials}

All chemicals were purchased from commercial suppliers and used without further purification: $\mathrm{Au}_{2} \mathrm{O}_{3}$ (99\%, ABCR), $\mathrm{Ag}_{2} \mathrm{O}$ (99\%, Sigma-Aldrich),
$\mathrm{HAuCl}_{4} \cdot 3 \mathrm{H}_{2} \mathrm{O}$ (Sigma-Aldrich), $\mathrm{AgNO}_{3}$ (99 \%, Alfa Aesar), oleylamine (technical grade, $70 \%$, Sigma), octylamine (for synthesis, Merck), dodecylamine (98\%, Aldrich), hexadecylamine (98\%, Aldrich), octadecylamine (98\%, Alfa Aesar), triphenylphosphine $(95 \%$, Fluka) and 1-undecanthiol (98\%, Aldrich). The solvents were of analytical grade quality and degassed by freeze-pomp-thaw technique prior to use: toluene, chlorobenzene, chloroform (ChemPur), decane, tetradecane (Aldrich) and 1,2-dichlorobenzene (ROTH). Silicon wafers were received from ITME (Warsaw). TEM grids were purchased from Ted Pella Inc.

Instrumentation

NMR spectra were recorded on Bruker (400 MHz) instrument. GC-MS analyses were performed on PerkinElmer Clarus 680/600S. MS spectra were recorded on Maldi SYNAPT G2-S HDMS (Waters) spectrometer. UV-Vis spectra were recorded using Evolution220 spectrophotometer from Thermo Scientific. XPS spectra were recorded on PHI 5000 VersaProbe X-ray photoelectron spectrometer using an Al KR X-ray source. FT-IR spectra were recorded on Jasco 6200 instrument. SEM, STEM and EDX were recorded on FEI Nova NanoSEM 450.

General procedure for the synthesis and characterization of NP dispersions

Metal precursor(s) $(0.01 \mathrm{mmol})$ and oleylamine ( $3 \mathrm{mmol}, 1 \mathrm{~mL}$ ) were loaded into $10 \mathrm{~mL}$ Schlenk tube. The tube was evacuated and filled with argon three times. If necessary, the degassed solvent was added. The tube was immersed into a pre-heated oil bath, and the suspension was stirred with $1 \mathrm{~cm}$ cylindrical bar at a speed of $1400 \mathrm{rpm}$. The samples for analyses were prepared as following. UV-Vis: aliquots of $1 \%(\mathrm{v} / \mathrm{v})$ of original volume were taken over the course of the reaction using automatic or glass pipette and diluted with chloroform; SEM, EDX and XPS: aliquots of $5 \%(\mathrm{v} / \mathrm{v})$ of original volume taken from the reaction mixture were diluted with $1: 1(\mathrm{v} / \mathrm{v})$ EtOH-MeOH and agitated on a laboratory mixer at $240 \mathrm{rpm}$ for $1 \mathrm{~h}$. If necessary, the dispersion was centrifuged at 2000-3500 rpm up to $10 \mathrm{~min}$ followed by the decantation of supernatant. The procedure was repeated up to 5 times. The NP sediment was dissolved 
in chloroform and deposited on a silicon wafer or TEM grid. GC-MS, IR, NMR and MS samples were obtained from the supernatant by evaporation of solvent on rotavap.

\section{Results and discussion}

We explored potential reductants of gold(III) oxide with a focus on substances capable of serving as ligands for AuNPs. The best results were obtained in organic media by using fatty amines. Other ligands, for example, thiols and phosphines produced polydisperse and aggregated NPs. The temperature regime was crucial to obtain high-quality NPs and secure them against aggregation. The reduction by amines required elevated temperatures $\left(>110^{\circ} \mathrm{C}\right)$ otherwise the reaction was sluggish furnishing NP aggregates. The NPs were also prone to aggregation at low metalamine ratios. The NP surface was not protected enough by amine ligands leading to uncontrolled growth and sintering of NPs. The effective passivation of NP surface was only attained at the ratios above 1:80. The correlation between the chain length of amine, and the size of NPs was not as apparent as for NPs derived from gold(III) chloride (Marchetti et al. 2011). The NP sizes were in range of $6-9 \mathrm{~nm}$ irrespective of aliphatic amine (C12-C18) used. In turn, the alkyl chain of octylamine was too short to effectively stabilize the metal core of NPs. The reaction proceeded equally well in aromatic and aliphatic hydrocarbon solvents that is consistent with previous reports on the reduction of gold(III) chloride (Wu et al. 2013). The reaction also took place without solvent, in particular when employing oleylamine. The latter was also superior in view of uniformity of NPs formed and therefore served us as a model to study the reduction of gold(III) oxide in more detail. The obtained nanoparticles are readily redispersible in nonpolar solvents, especially in chloroform and can be further functionalized with organic thiols (1-undecanthiol, 11-mercaptoundecyl- $N, N, N$-trimethylammonium bromide).

In a typical experiment, the suspension of gold(III) oxide and an excess of oleylamine was vigorously stirred upon heating. The reaction was performed in neat amine (300 equiv.) under a blanket of argon. Increasing the initial amount of oleylamine (up to 1200 equiv.) had almost no influence both on the reaction time and the size of NPs. The use of protective inert gas atmosphere was necessary as the NPs were aggregated in air. During the reaction, the color of the solution gradually turned deep red indicating the formation of NPs. The appearance of surface plasmon resonance band enabled to follow the process by UVVis spectroscopy. The corresponding time-resolved spectra are shown in Fig. 1. For $130{ }^{\circ} \mathrm{C}$, the plasmonic peak was increased over $24 \mathrm{~h}$ indicating the continuous formation of AuNPs. At higher temperature $\left(180^{\circ} \mathrm{C}\right)$, the reaction was virtually completed within 15 min. In both cases, the absorption maximum drifted slightly over time toward the red end of the spectrum due to NP growth. SEM imaging showed that the initially formed NPs were suffered from the broad size distribution (Fig. S1). This is not surprising taking into account the heterogeneity of the feed mixture. However, the NPs became more uniform upon prolonged heating. The best quality (in terms of dispersity) NPs were obtained at $180{ }^{\circ} \mathrm{C}$ after $24 \mathrm{~h}$. The micrographs and size distribution of NPs are depicted in Fig. 2. The low polydispersity is also nicely manifested by the hexagonal arrangement of NPs as seen in low-magnification SEM images (Fig. S2). The XPS analysis revealed that the core of NPs consists of metallic gold. The binding energy for Au4f $\mathrm{f}_{7 / 2}$ band $(84.4 \mathrm{eV})$ is in good agreement with literature data for $\mathrm{Au}^{0}$ (Casaletto et al. 2006). No signals corresponding to oxidized gold species were observed indicating the quantitative formation of gold nanoparticles. The surface of NPs is covered by neutral amine ligands as evidenced by the presence of a single peak at $398.8 \mathrm{eV}$ (Kumar et al. 2003). XPS spectra are shown in Fig. 3.

It should be noted that gold(III) oxide is thermally unstable compound which spontaneously decomposes to gold and oxygen. To define unambiguously the function of amine in the process of NP formation, the gold precursor was heated without amine additive using chlorobenzene as a solvent. The experiment was conducted at $130{ }^{\circ} \mathrm{C}$ for $6 \mathrm{~h}$. Neither the formation of NPs nor changes in the morphology of gold(III) oxide was noticed by SEM (Fig. S3). Nonetheless, the loss of oxygen from the sample was revealed by EDX. The rate of the spontaneous deoxygenation of gold(III) oxide, however, was much slower than that in the presence of amine. That is, the amine serves not only as a ligand but also as a reducing agent in the process. The reduction probably occurs through an electron 

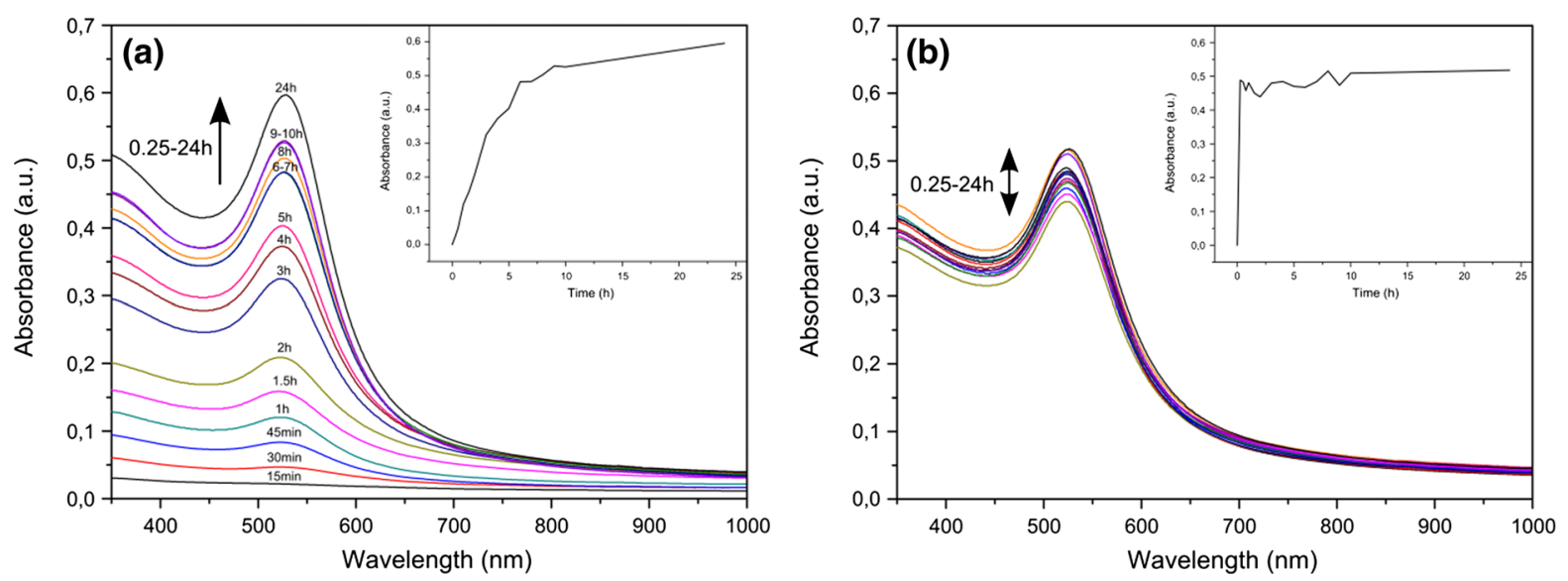

Fig. 1 UV-Vis traces of the formation of AuNPs at $130{ }^{\circ} \mathrm{C}(\mathbf{a})$ and $180{ }^{\circ} \mathrm{C}(\mathbf{b})$. The insets show the absorbance as a function of time at $\lambda=526 \mathrm{~nm}$
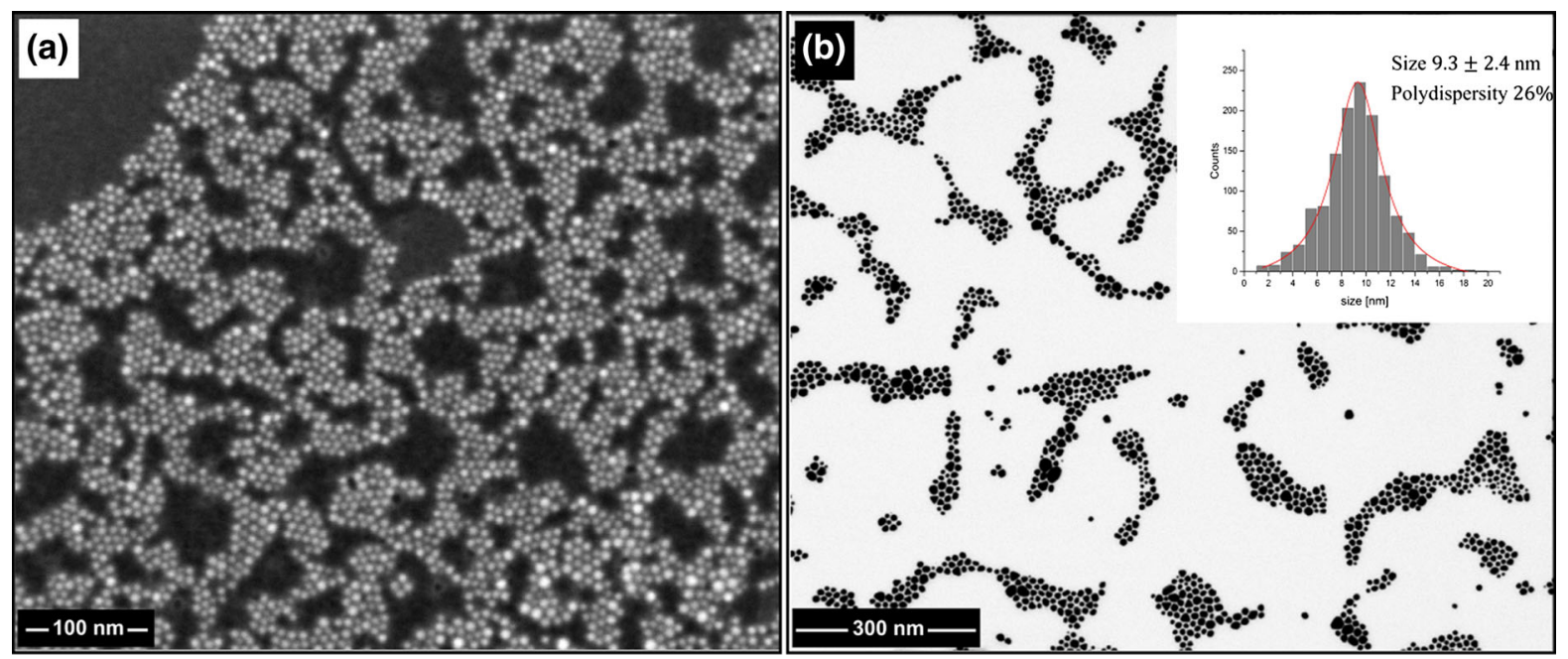

Fig. 2 SEM (a) and STEM (b) micrographs of NPs obtained in oleylamine at $180{ }^{\circ} \mathrm{C}, 24 \mathrm{~h}$. The inset shows the NP size distribution

transfer to gold ions that lead to the oxidation of amine to nitrile (Chen et al. 2007; Liu et al. 2007). For the sake of simplicity, octylamine was employed in mechanistic studies. Indeed, the presence of trace amounts of octanenitrile was detected by GC-MS (Fig. S4). Besides, the more abundant signals belonging to unidentified compounds with higher mass and amide like profile were observed. A new band at $1646 \mathrm{~cm}^{-1}$ that likely corresponds to stretching mode of either $\mathrm{C}=\mathrm{O}$ or $\mathrm{C}=\mathrm{N}$ bond was found in FT-IR spectrum (Fig. 4a). The peaks characteristic for amide and amidine moieties was also observed in ${ }^{1} \mathrm{H}$ NMR spectrum (Fig. 4b). Further inspection by ${ }^{13} \mathrm{C}$ NMR spectroscopy confirmed the presence of two different compounds containing $\mathrm{C}=\mathrm{X}$ bond (where $\mathrm{X}$ is $\mathrm{O}$ or $\mathrm{N}$ ).
ESI-MS analysis (Fig. S5-S6) ascribed eventually the observed NMR shifts to $N$-octyloctanamide (4) and $N, N$ '-dioctyloctanimidamide (5). The products are derivatives of $\mathrm{N}$-octyloctanimidamide (3) which is formed in the reaction of nitrile (2) with an excess of amine (1) present in the solution. The fate of amine during the preparation of AuNPs is presented in Fig. 5.

The method of NP preparation is not only limited to gold but can also be extended to other metals. For example, the reduction of silver(I) oxide to silver nanoparticles took place under conditions outlined above (Fig. S7). This fact prompted us to investigate the influence of chloride ions on the formation of NPs in binary $\mathrm{Au}-\mathrm{Ag}$ mixtures (Fig. 6). A trend toward decreasing the size of NPs was also maintained in this 


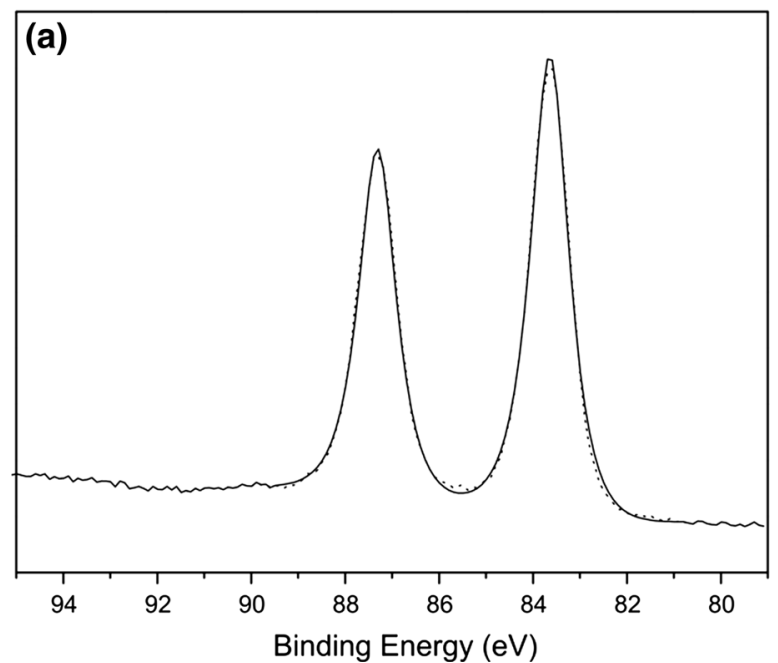

Fig. 3 XPS evidence for the formation of amine-capped AuNPs. The experimental and fitted data are depicted as dotted and solid lines, respectively. The single components in Au4f

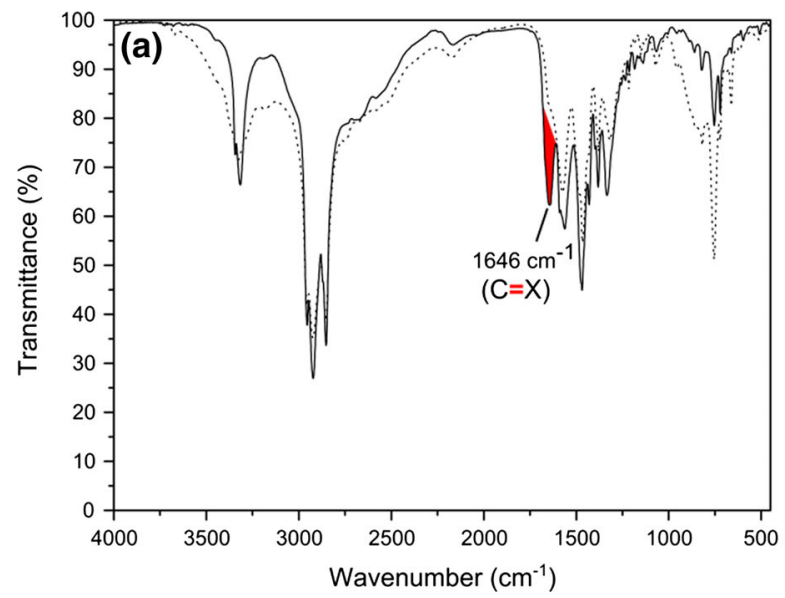

Fig. 4 Partial IR (a) and NMR (b) spectra of postreaction mixture obtained by heating gold(III) oxide with octylamine. The reaction was performed at $110{ }^{\circ} \mathrm{C}$ in toluene- $\mathrm{d}_{8}$ for $24 \mathrm{~h}$. IR

case. The NPs obtained by co-reduction of gold and silver oxides were significantly smaller than those obtained from chloroauric acid and silver nitrate (Fig. 6b). The difference was also observed in the composition of the NPs. The NPs obtained in the presence of chloride ions had a lowered content of silver due to the precipitation of silver chloride (Fig. S8). Furthermore, the structure of these NPs seems to be inhomogeneous with a core and a shell enriched in gold and silver, respectively. This is indicated by the shift of plasmonic band toward shorter wavelength despite the precipitation of $\mathrm{AgCl}$ (Fig. 6a). There is also an

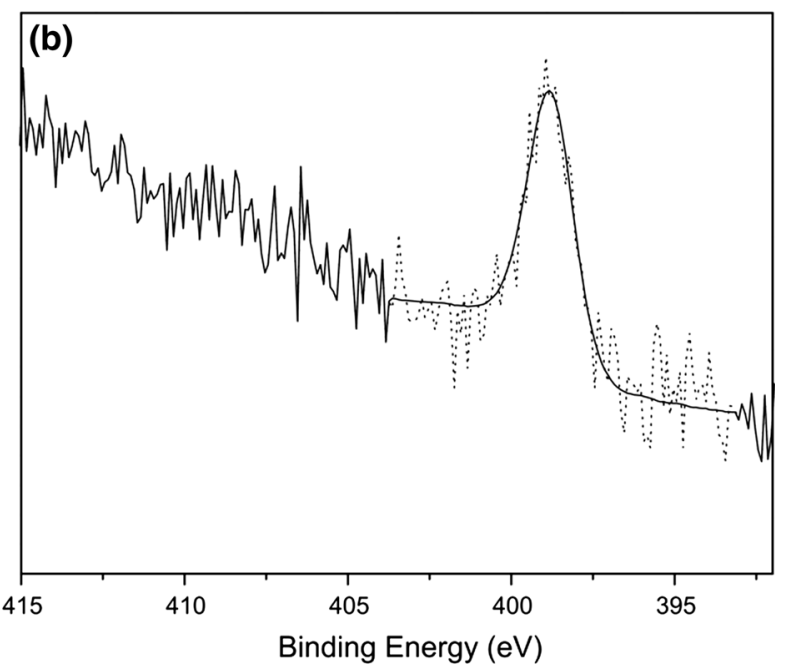

(a) and N1s (b) regions correspond to the reduced gold $\left(\mathrm{Au}^{0}\right)$ and non-protonated amine group $\left(-\mathrm{NH}_{2}\right)$, respectively

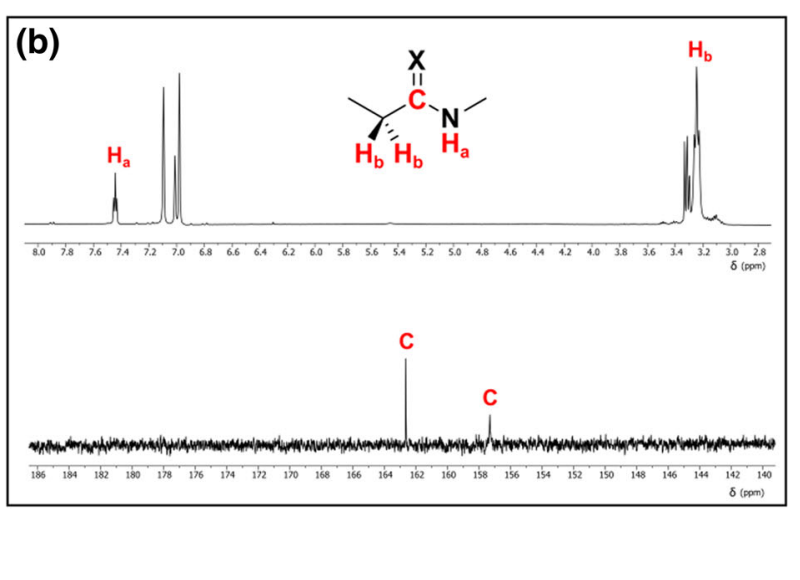

spectrum of pure octylamine is represented as a dotted line. The signals characteristic for amide and amidine group are marked in red. (Color figure online)

apparent shoulder near $500 \mathrm{~nm}$ that might be diagnostic of the separation of metal phases (Lu et al. 2013; Zeng et al. 2014). The generation of gold seeds was observed visually by the appearance of red color shortly after the reaction was started. The color next rapidly changed through brown (reduction of $\mathrm{Ag}^{+}$) to dark (sedimentation of the NPs). The observed reduction sequence has been described previously (Wang et al. 2009). Conversely, the NPs obtained from metal oxides appears to be efficiently alloyed retaining $1: 1 \mathrm{Au}-\mathrm{Ag}$ feed ratio as evidenced by plasmonic peak centered at $473 \mathrm{~nm}$ (Rajendra et al. 2015). 


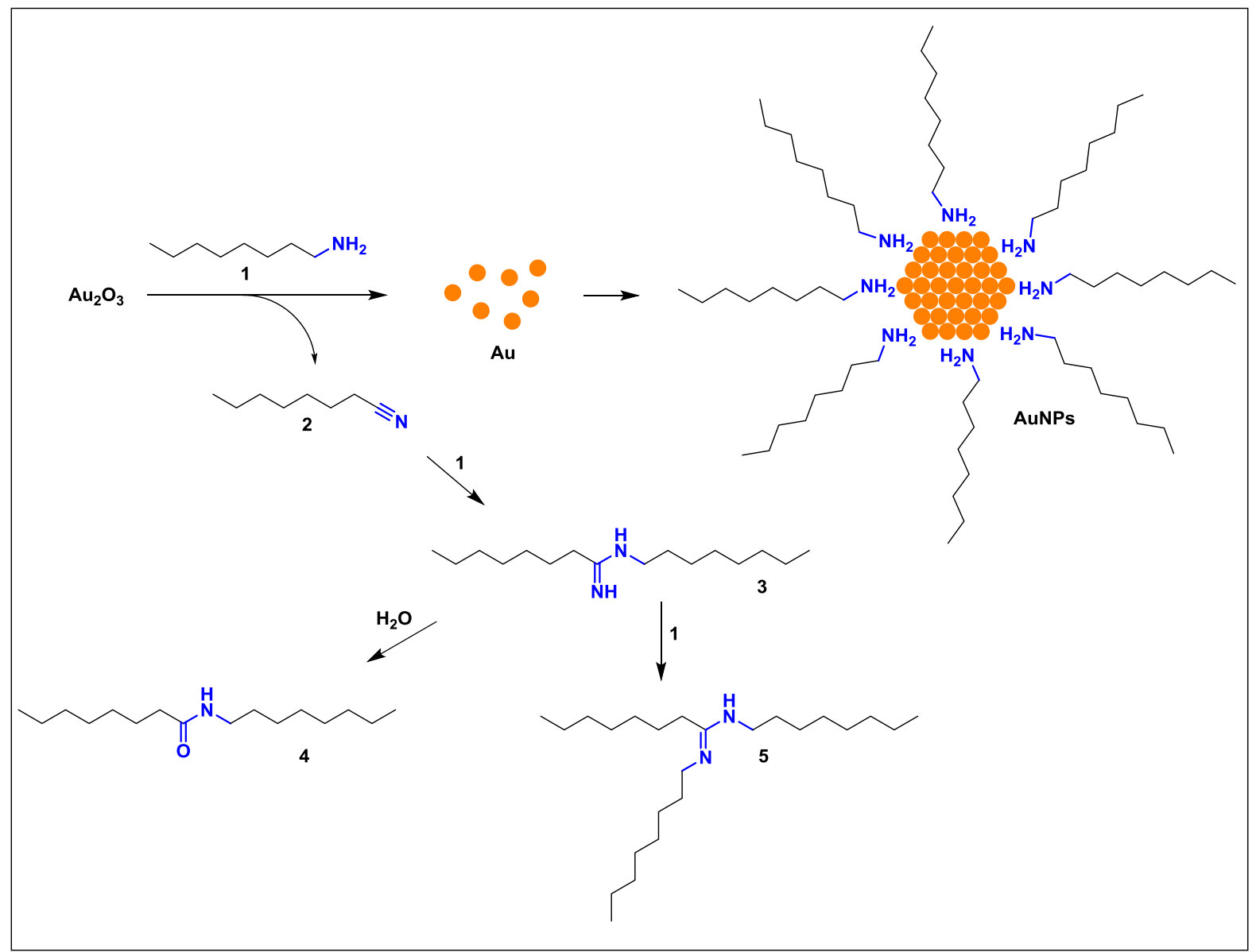

Fig. 5 Cartoon representation of chemical processes that take place during the NP formation. The amine 1 reduces gold(III) oxide to gold and stabilizes the surface of AuNPs. The nitrile 2 which is the product of amine oxidation reacts with the amine $\mathbf{1}$ to give amidine derivative $\mathbf{3}$. The adduct $\mathbf{3}$ undergoes next hydrolysis and aminolysis to afford $\mathbf{4}$ and $\mathbf{5}$, respectively (a)

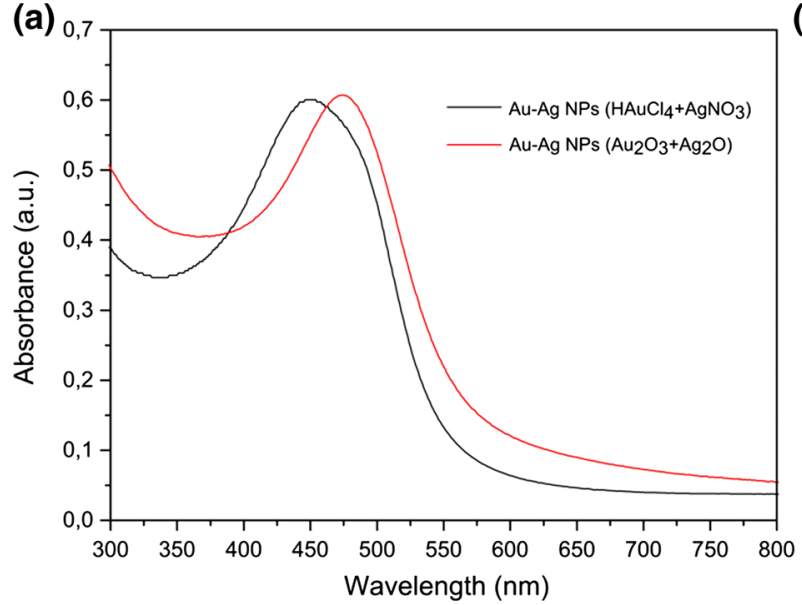

(b)

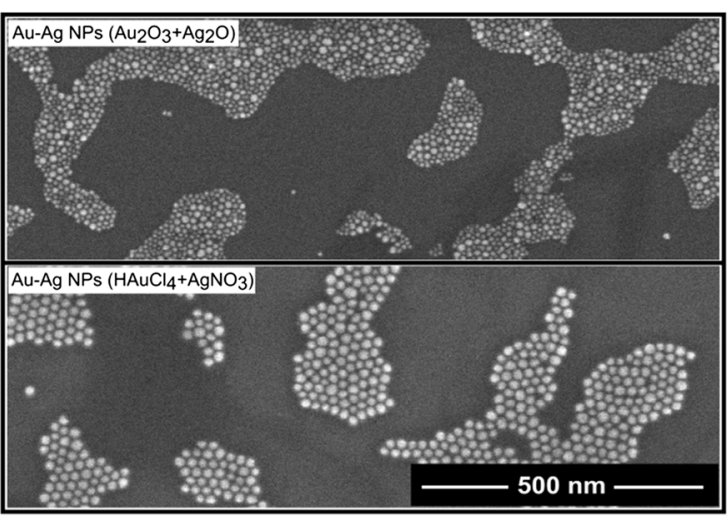

Fig. $6 \mathrm{UV}-\mathrm{Vis}(\mathbf{a})$ and SEM (b) traces of $\mathrm{Au}-\mathrm{Ag}$ NPs obtained in the presence and absence of chloride ions. In both cases, the reaction was performed with the same concentrations of $\mathrm{Au}$ and $\mathrm{Ag}$ precursors $(0.01 \mathrm{M})$ in bulk amine at $180{ }^{\circ} \mathrm{C}$ for $3 \mathrm{~h}$ 


\section{Conclusions}

In summary, we developed a new method for obtaining noble metal NPs from corresponding oxides by reducing with aliphatic amines. The NPs obtained by this method are usually smaller than those prepared using halogen-containing metal precursors. For example, the reduction of gold(III) oxide yields sub-10 nm particles with good monodispersity. The lack of halogen has also influence on the final composition of NPs. The NPs made of gold and silver are alloyed better than those obtained in the presence of halogen. Finally, and importantly, the presented approach adheres well to green chemistry principles. The synthesis is performed in the absence of organic solvents and the reagents used are non-toxic (gold(III) oxide) and manufactured from natural oils (oleylamine).

Acknowledgments This project was funded by National Science Center (Grant Sonata UMO-2011/01/D/ST5/03518). We thank Grzegorz Sobczak and Tomasz Wojciechowski for measurements of some SEM and EDX data.

Open Access This article is distributed under the terms of the Creative Commons Attribution 4.0 International License (http:// creativecommons.org/licenses/by/4.0/), which permits unrestricted use, distribution, and reproduction in any medium, provided you give appropriate credit to the original author(s) and the source, provide a link to the Creative Commons license, and indicate if changes were made.

\section{References}

Aslam M, Fu L, Su M, Vijayamohanan K, Dravid VP (2004) Novel one-step synthesis of amine-stabilized aqueous colloidal gold nanoparticles. J Mater Chem 14:1795-1797. doi:10.1039/B402823F

Bhargava SK, Booth JM, Agrawal S, Coloe P, Kar G (2005) Gold nanoparticle formation during bromoaurate reduction by amino acids. Langmuir 21:5949-5956. doi:10.1021/ la050283e

Brust M, Walker M, Bethell D, Schiffrin DJ, Whyman R (1994) Synthesis of thiol-derivatised gold nanoparticles in a twophase liquid-liquid system. J Chem Soc Chem Commun. doi:10.1039/C39940000801

Casaletto MP, Longo A, Martorana A, Prestianni A, Venezia AM (2006) XPS study of supported gold catalysts: the role of $\mathrm{Au} 0$ and $\mathrm{Au}+\delta$ species as active sites. Surf Interface Anal 38:215-218. doi:10.1002/sia.2180

Chen M, Feng Y-G, Wang X, Li T-C, Zhang J-Y, Qian D-J (2007) Silver nanoparticles capped by oleylamine: formation, growth, and self-organization. Langmuir 23:5296-5304. doi:10.1021/1a700553d
Daniel M-C, Astruc D (2004) Gold nanoparticles: assembly, supramolecular chemistry, quantum-size-related properties, and applications toward biology, catalysis, and nanotechnology. Chem Rev 104:293-346. doi:10.1021/ cr030698+

Dozol H, Mériguet G, Ancian B, Cabuil V, Xu H, Wang D, Abou-Hassan A (2013) On the synthesis of Au nanoparticles using EDTA as a reducing agent. J Phys Chem C 117:20958-20966. doi:10.1021/jp4067789

Fanizza E, Depalo N, Clary L, Agostiano A, Striccoli M, Curri ML (2013) A combined size sorting strategy for monodisperse plasmonic nanostructures. Nanoscale 5:3272-3282. doi:10.1039/C3NR33944K

Giljohann DA, Seferos DS, Daniel WL, Massich MD, Patel PC, Mirkin CA (2010) Gold nanoparticles for biology and medicine. Angew Chem Int Ed 49:3280-3294. doi:10. 1002/anie.200904359

Hakkinen H (2012) The gold-sulfur interface at the nanoscale. Nat Chem 4:443-455. doi:10.1038/NCHEM.1352

Hiramatsu H, Osterloh FE (2004) A simple large-scale synthesis of nearly monodisperse gold and silver nanoparticles with adjustable sizes and with exchangeable surfactants. Chem Mater 16:2509-2511. doi:10.1021/cm049532v

Jana NR, Gearheart L, Murphy CJ (2001) Seeding growth for size control of 5-40 nm diameter gold nanoparticles. Langmuir 17:6782-6786. doi:10.1021/la0104323

Jana NR, Peng X (2003) Single-phase and gram-scale routes toward nearly monodisperse $\mathrm{Au}$ and other noble metal nanocrystals. J Am Chem Soc 125:14280-14281. doi:10. 1021/ja038219b

Jans H, Huo Q (2012) Gold nanoparticle-enabled biological and chemical detection and analysis. Chem Soc Rev 41:2849-2866. doi:10.1039/C1CS15280G

Kale MJ, Avanesian T, Christopher P (2014) Direct photocatalysis by plasmonic nanostructures. ACS Catal 4:116-128. doi:10.1021/cs400993w

Kalsin AM, Fialkowski M, Paszewski M, Smoukov SK, Bishop KJM, Grzybowski BA (2006) Electrostatic self-assembly of binary nanoparticle crystals with a diamond-like lattice. Science 312:420-424. doi:10.1126/science.1125124

Kimling J, Maier M, Okenve B, Kotaidis V, Ballot H, Plech A (2006) Turkevich method for gold nanoparticle synthesis revisited. J Phys Chem B 110:15700-15707. doi:10.1021/ jp061667w

Kumar A, Mandal S, Pasricha R, Mandale AB, Sastry M (2003) Investigation into the interaction between surface-bound alkylamines and gold nanoparticles. Langmuir 19:6277-6282. doi:10.1021/1a034209c

Lakshminarayana P, Qing-Hua X (2009) A simple method for large scale synthesis of highly monodisperse gold nanoparticles at room temperature and their electron relaxation properties. Nanotechnology 20:185606

Lee Y, Loew A, Sun S (2010) Surface- and structure-dependent catalytic activity of Au nanoparticles for oxygen reduction reaction. Chem Mater 22:755-761. doi:10.1021/ cm9013046

Leff DV, Brandt L, Heath JR (1996) Synthesis and characterization of hydrophobic, organically-soluble gold nanocrystals functionalized with primary amines. Langmuir 12:4723-4730. doi:10.1021/la960445u 
Li W, Sun Q, Su B (2013) Effect of chloride anion on the electrochemical charging of gold nanoparticle films. J Solid State Electrochem 17:2429-2435. doi:10.1007/ s10008-013-2121-4

Liu X, Atwater M, Wang JH, Dai Q, Zou JH, Brennan JP, Huo Q (2007) A study on gold nanoparticle synthesis using oleylamine as both reducing agent and protecting ligand. J Nanosci Nanotechnol 7:3126-3133. doi:10.1166/jnn. 2007.805

Lu L, Burkey G, Halaciuga I, Goia DV (2013) Core-shell gold/ silver nanoparticles: synthesis and optical properties. J Colloid Interface Sci 392:90-95. doi:10.1016/j.jcis.2012. 09.057

Marchetti B, Joseph Y, Bertagnolli H (2011) Amine-capped gold nanoparticles: reaction steps during the synthesis and the influence of the ligand on the particle size. J Nanopart Res 13:3353-3362. doi:10.1007/s11051-011-0358-3

Martin MN, Basham JI, Chando P, Eah S-K (2010) Charged gold nanoparticles in non-polar solvents: 10-min synthesis and 2D self-assembly. Langmuir 26:7410-7417. doi:10. 1021/la100591h

Merga G, Saucedo N, Cass LC, Puthussery J, Meisel D (2010) "Naked" gold nanoparticles: synthesis, characterization, catalytic hydrogen evolution, and SERS. J Phys Chem C 114:14811-14818. doi:10.1021/jp104922a

Mikami Y, Dhakshinamoorthy A, Alvaro M, Garcia H (2013) Catalytic activity of unsupported gold nanoparticles. Catal Sci Tech 3:58-69. doi:10.1039/C2CY20068F

Mourdikoudis S, Liz-Marzán LM (2013) Oleylamine in nanoparticle synthesis. Chem Mater 25:1465-1476. doi:10. 1021/cm4000476

Nealon GL, Donnio B, Greget R, Kappler J-P, Terazzi E, Gallani J-L (2012) Magnetism in gold nanoparticles. Nanoscale 4:5244-5258. doi:10.1039/C2NR30640A

Perrault SD, Chan WCW (2009) Synthesis and surface modification of highly monodispersed, spherical gold nanoparticles of 50-200 nm. J Am Chem Soc 131:17042-17043. doi:10.1021/ja907069u

Rai A, Singh A, Ahmad A, Sastry M (2006) Role of halide ions and temperature on the morphology of biologically synthesized gold nanotriangles. Langmuir 22:736-741. doi:10.1021/la052055q

Rajendra R, Bhatia P, Justin A, Sharma S, Ballav N (2015) Homogeneously-alloyed gold-silver nanoparticles as per feeding moles. J Phys Chem C 119:5604-5613. doi:10. $1021 / j p 512528 j$

Rak MJ, Saade NK, Friscic T, Moores A (2014) Mechanosynthesis of ultra-small monodisperse amine-stabilized gold nanoparticles with controllable size. Green Chem 16:86-89. doi:10.1039/C3GC41827H

Saha K, Agasti SS, Kim C, Li X, Rotello VM (2012) Gold nanoparticles in chemical and biological sensing. Chem Rev 112:2739-2779. doi:10.1021/cr2001178

Sashuk V (2012) Thiolate-protected nanoparticles via organic xanthates: mechanism and implications. ACS Nano 6:10855-10861. doi:10.1021/nn304229r

Sashuk V, Hołyst R, Wojciechowski T, Górecka E, Fiałkowski M (2012) Autonomous self-assembly of ionic nanoparticles into hexagonally close-packed lattices at a planar oil- water interface. Chem Eur J 18:2235-2238. doi:10.1002/ chem.201103272

Sashuk V, Winkler K, Żywociński A, Wojciechowski T, Górecka E, Fiałkowski M (2013) Nanoparticles in a capillary trap: dynamic self-assembly at fluid interfaces. ACS Nano 7:8833-8839. doi:10.1021/nn403297f

Scarabelli L, Coronado-Puchau M, Giner-Casares JJ, Langer J, Liz-Marzán LM (2014) Monodisperse gold nanotriangles: size control, large-scale self-assembly, and performance in surface-enhanced Raman scattering. ACS Nano 8:5833-5842. doi:10.1021/nn500727w

Shen C et al (2008) Monodisperse noble-metal nanoparticles and their surface enhanced Raman scattering properties. Chem Mater 20:6939-6944. doi:10.1021/cm800882n

Singh S, Pasricha R, Bhatta UM, Satyam PV, Sastry M, Prasad BLV (2007) Effect of halogen addition to monolayer protected gold nanoparticles. J Mater Chem 17:1614-1619. doi:10.1039/B614934K

Stratakis M, Garcia H (2012) Catalysis by supported gold nanoparticles: beyond aerobic oxidative processes. Chem Rev 112:4469-4506. doi:10.1021/cr3000785

Teulle A, Bosman M, Girard C, Gurunatha KL, Li M, Mann S, Dujardin E (2015) Multimodal plasmonics in fused colloidal networks. Nat Mater 14:87-94. doi:10.1038/ nmat 4114

Wang C, Yin H, Chan R, Peng S, Dai S, Sun S (2009) One-pot synthesis of oleylamine coated AuAg alloy NPs and their catalysis for CO oxidation. Chem Mater 21:433-435. doi:10.1021/cm802753j

Wells DM, Rossi G, Ferrando R, Palmer RE (2015) Metastability of the atomic structures of size-selected gold nanoparticles. Nanoscale 7:6498-6503. doi:10.1039/ C4NR05811A

Wu B-H, Yang H-Y, Huang H-Q, Chen G-X, Zheng N-F (2013) Solvent effect on the synthesis of monodisperse aminecapped Au nanoparticles. Chin Chem Lett 24:457-462. doi:10.1016/j.cclet.2013.03.054

Yeh Y-C, Creran B, Rotello VM (2012) Gold nanoparticles: preparation, properties, and applications in bionanotechnology. Nanoscale 4:1871-1880. doi:10.1039/ C1NR11188D

Yu Y, Yang W, Sun X, Zhu W, Li XZ, Sellmyer DJ, Sun S (2014) Monodisperse MPt ( $\mathrm{M}=\mathrm{Fe} \mathrm{Co}, \mathrm{Ni}, \mathrm{Cu}, \mathrm{Zn}$ ) nanoparticles prepared from a facile oleylamine reduction of metal salts. Nano Lett 14:2778-2782. doi:10.1021/ n1500776e

Zeng JB et al (2014) Au@ Ag core/shell nanoparticles as colorimetric probes for cyanide sensing. Nanoscale 6:9939-9943. doi:10.1039/C4NR02560A

Zhang J, Xi C, Feng C, Xia H, Wang D, Tao X (2014) High yield seedless synthesis of high-quality gold nanocrystals with various shapes. Langmuir 30:2480-2489. doi:10.1021/ la404602h

Zhao P, Li N, Astruc D (2013) State of the art in gold nanoparticle synthesis. Coord Chem Rev 257:638-665. doi:10.1016/j.ccr.2012.09.002

Zhao H et al (2016) Reversible trapping and reaction acceleration within dynamically self-assembling nanoflasks. Nat Nanotechnol 11:82-88. doi:10.1038/nnano.2015.256 